\title{
Information, Interaktion, Innovation ... ein informationspraktisches Editorial
}

Liebe Mitglieder und Freunde der DGI,

zwei intensive, von der DGI geprägte Tage liegen hinter mir, mit Licht und Schatten - aber doch am Ende einem guten Gefühl.

Da war zum einen der gestern, am 8. November, von Clemens Weins moderierte Workshop zum Thema „Die DGI der Zukunft - Entwicklung unserer gemeinnützigen Fachgesellschaft“, in dem eine relativ überschaubare, deshalb aber auch wirklich zur Diskussion fähige Gruppe von Mitgliedern recht bald an einen Punkt kam, an dem die strategischen Alternativen für die Weiterentwicklung der DGI sichtbar wurden: wollen wir uns in Zukunft als Lobbyverband sehen (aber dann welcher Berufsgruppe verpflichtet?) - oder doch weiter als eine Fachgesellschaft, die Information Professionals unterschiedlicher Couleur zusammenbringt, die ein Interesse an den Kernbegriffen ,Wissen' und ,Information' gemeinsam haben, ohne dass diese Fachgesellschaft dabei dem partikularen Interesse eines Berufsstandes verpflichtet wäre. Dabei wurde deutlich, dass die erste Alternative sich im Grunde schon deshalb fast verbietet, weil es eine homogene Interessengruppe, für die eine solche Lobbyorganisation tätig sein könnte, nicht (mehr) gibt. Wir möchten uns also als Fachgesellschaft weiter entwickeln, dabei jedoch die Gewichte deutlich verschieben zugunsten der Informationspraxis - wenn auch selbstverständlich mit informationswissenschaftlicher Unterfütterung.

Diesen Konsens spiegelte auch die sich anschließende Diskussion um eine mögliche Umbenennung der DGI wider - wobei der von allen betonte Ausgangspunkt die möglichste Rettung des Akronyms war. Vor allem aber kürzer sollte der Name sein, und da zudem eine Benennung als „Deutsche Gesellschaft für Wissen“ denn doch sehr prätentiös gewesen wäre einigten wir uns schließlich auf „Deutsche Gesellschaft für Information e. V.“ mit dem Untertitel „in Wissenschaft und Praxis“.

Diesen Vorschlag trugen wir dann auch in die anschließende außerordentliche Mitgliederversammlung, die ihn im Prinzip begrüßte - jedoch für einen Vorschlag zur Beschlussfassung doch einigen sprachlich-grammatischen Nachbesserungsbedarf sah. Doch war dies nicht das Hauptthema der Versammlung: nötig war sie vor allem geworden, weil die wirklich Besorgnis erregende finanzielle Lage der DGI uns als Vorstand gezwungen hatte, eine Beitragserhöhung vorzuschlagen, der die Mitglieder glücklicherweise auch nach einer nicht unkontroversen Aussprache zustimmten. Die Handlungsfähigkeit der DGI und insbesondere unserer Geschäftsstelle bleibt damit erhalten - ist aber weiter gefährdet!

Die damit geworfenen Schatten wurden dann aber rasch wieder aufgehellt durch das anschließende „Kamingespräch“, in dem fünf sehr kundige Disputanten unter der Moderation von Clemens Weins unter dem Titel „Vom wertvollsten Gut zum nervtötenden Zeitfresser ... Viel Information | Wenig Kompetenz?“ eine sehr unterhaltsame Diskussion vor allem über soziale und interaktive Technologien im Internet und über die mit diesen verbundenen Hoffnungen und Bedrohungen führten.

Diese in dieser Runde zusammengebrachte wirklich gelungene Mischung aus Politik und Sachverstand in Kombination mit den großenteils auf sehr hohem Niveau befindlichen Vorträgen des dann heute folgenden zweiten Tages unserer zweiten „Praxistage“ bestätigten im Nachhinein noch einmal den im Workshop am Vortag gefundenen Konsens: in der Praxisarbeit der DGI und ihrer dort vertretenen Mitglieder steckt so viel sehr plastisch darstellbares Potential, dass am Ende wirklich so etwas wie Aufbruchsstimmung entstanden war.

Den Titel dieser Zeitschrift werden wir deshalb nicht ändern: sie bleibt primär dem informationswissenschaftlichen Aspekt der DGI verbunden. Doch in der Verbandsarbeit der DGI sollte eine geänderte Reihenfolge von „Praxis“ und „Wissenschaft“ die geänderte Priorisierung widerspiegeln.

Eines allerdings muss dabei ganz deutlich werden: bei dieser Entwicklung handelt es sich mitnichten um eine regressive Bewegung zurück in die glorreiche Ära der Informationsvermittlung! Das mit dieser Vergangenheit verbundene Tätigkeitsfeld ist im Absterben begriffen und vielerorts auch obsolet: identitätskonstitutiv kann es nicht mehr sein. Sehr wohl aber kann eine praxisbezogene Hinwendung zu den neuen Modalitäten des inhaltlichen und technischen Umgangs mit Information und Wissen im Web eine neue, nunmehr praxisfundierte Identität der DGI begründen. Dies Potential wurde bei den Praxistagen sichtbar: lassen Sie uns es heben!

In diesem Sinne wünsche ich Ihnen eine gute Lektüre des vorliegenden Heftes und einen gesunden Start in ein glückliches und erfolgreiches Neues Jahr für uns alle!

Herzliche Grüße - Ihr Stefan Gradmann 


\section{Manfred Bonitz (7.3.1931-14.8.2012)}

Dr. Manfred Bonitz gehörte zu den Pionieren der Informationswissenschaft in Deutschland. Geboren 1931 in Chemnitz, studierte er zunächst Physik in der DDR und der Sowjetunion. Seine Habilitationsschrift über „Kernspektroskopie am Deuteronenstrahl” verteidigte er 1969 an der Technischen Universität Dresden, Sektion Physik. Sein Weg in die Informationswissenschaft ist typisch für viele Physiker nach dem Zweiten Weltkrieg in Ost und West. Man denke zum Beispiel an den amerikanischen Informationswissenschaftler Derek de Solla Price.

Eine Bibliographie von Manfred Bonitz' wissenschaftlichen Schriften weist für 1970 eine erste 'informationswissenschaftliche' Publikation aus: „Das Internationale Nuklear-Informationssystem INIS. - ZfK-194. Rossendorf: Zentralinstitut für Kernforschung 1970.” [1] Wie im Web of Science ${ }^{\circledR}$ sichtbar erscheinen seine Publikationen seit den 1970er Jahren nicht mehr in Fachzeitschriften wie Nuclear Instruments \& Methods und Nuclear Physics A, sondern dem International Forum on Information and Documentation und vor allem in Scientometrics. Ein leidenschaftlicher, experimenteller Forscher blieb Manfred auch in seinem neuen Gebiet - der Informationswissenschaft. Darum galt der Scientometrics oder Wissenschaftsmetrie - als der quantitativen Wissenschaftsforschung - sein ganz besonderes Interesse. Mit Eugene Garfield, dessen Citation Indexing die empirische Grundlage dieses Gebietes schuf, verband ihn eine enge, langjährige Freundschaft. Vasili Vassilievich Nalimov, dem Namensgeber dieses Gebiet, widmete er seine Kraft in den letzten Jahren, als Übersetzer und Herausgeber seiner Werke.

Manfred Bonitz' Streben nach wissenschaftlicher Fundierung dieses relativ neuen Gebietes fand einen Höhepunkt in seinen eignen Arbeiten zur empirischen Validierung von Mertons berühmtem ,Matthäus-Effekts in der Wissenschaft‘. In Kurzfassung: Manfred Bonitz analysierte das Publikationsaufkommen ganzer Länder und konnte zeigen, dass es eine systematische Abweichung zwischen den Erwartungswerten von Zitationen (basierend auf dem Impact Faktor von Zeitschriften, in denen Wissenschaftler mit Adressen in diesen Ländern publizieren) und der tatsächlichen Anzahl von Zitationen gibt. Eine kleine Gruppe von zumeist hochindustrialisierten Ländern ist deutlicher Gewinner in dem ,Spiel“ der Verteilung von Zitationen über Publikationen. Noch immer werden seine Arbeiten zum Matthäuseffekt zitiert, in jüngerer Zeit vor allem von Physikern (!), die sich der Netzwerkanalyse von Zitationsnetzwerken verschreiben.

Aber es wäre zu kurz gegriffen, Manfred Bonitz' Wirken auf die Wissenschaftsmetrie allein $\mathrm{zu}$ beschränken.
Er gehörte zu der Generation, die am Anfang des definitorischen Ringens um Begriffe wie: Information, Informatik (Informatika im Russischen oder Informatics im Englischen) und Kybernetik in der Informationswissenschaft stand. Wie wenige andere Wissenschaftler war er sowohl mit den Diskursen der Information- und Dokumentationsspezialisten, als auch der Wissenschaftsmetriker bekannt. Er ,wanderte' zwischen Kongressen wie dem Deutschen Dokumentartag, der International Society for Knowledge Organization, und der International Society of Scientometrics and Informetrics und wusste, wie wenige andere, diese inzwischen zum Teil auseinander strebenden Welten miteinander zu verbinden. Vor allem aber wirkte er ganz konkret: forschend, aufklärend und verbindend. Sein Anliegen war es Grundlagenforschung auf internationalem Niveau mit den praktischen Informations-Aufgaben von Instituten zu verbinden.

Seine Einsichten in das Wesen von wissenschaftlicher Information haben in seinem noch immer lesenswerten Buch „Wissenschaftliche Forschung und wissenschaftliche Information“ (erschienen in der Reihe ,Beiträge zur Forschungstechnologie“ des Akademie Verlages Berlin 1981) komprimierten Ausdruck gefunden. Dieses Buch hat Generationen von Informationswissenschaftlern geprägt, nicht nur in Deutschland, wie ich kürzlich von einem Kollegen in den Niederlanden hörte.

Die von ihm formulierten „Verhaltensprinzipien wissenschaftlichen Information“ (Holographie- und Tempoprinzip) und deren Konsequenz für die tägliche Informationssuche im Zeitalter von Digitalisierung und Internet sind weiterhin des Nachdenkens und Untersuchens wert.

Manfred Bonitz war nicht nur ein beseelter Forscher, er war auch ein gütiger und liebenswerter Mensch. Vielleicht waren es die Kindheitserlebnisse in der Zeit von Faschismus und Krieg, die seine humanistische Grundeinstellung prägten und ihn immer wieder Brücken bauen ließen: zwischen Ost und West; zwischen Deutschland und der Sowjetunion/Russland und zwischen verschiedenen Fachgebieten. Dass sich Informationswissenschaftler aller Couleur wieder auf gemeinsame Wurzeln besinnen, ist wohl die wichtigste Botschaft dieser Generation an uns.

\section{Andrea Scharnhorst, Amsterdam, Berlin}

[1] Bibliographie Manfred Bonitz. Zusammengestellt van Heinrich Parthey anlässlich des 70sten Geburtstages von M. Bonitz. In: Wissenschaftliche Zeitschrift und Digitale Bibliothek. Wissenschaftsforschung Jahrbuch 2002. Hrsg. v. Heinrich Parthey, Walter Umstätter. Gesellschaft für Wissenschaftsforschung, Berlin 2003, 2. Aufl. 2011, Seiten 175-191. Online verfügbar: http:// www.wissenschaftsforschung.de/Jahrbuch2002.pdf 